\title{
EFFECTS OF DROUGHT STRESS ON TASSEL GROWTH AND CHARACTERISTICS PHYSIOLOGICAL OF MAIZE VARIETIES
}

\author{
LI, J. ${ }^{1,2 \dagger}-$ WANG, T. ${ }^{1,2 \dagger}-$ WANG, C. ${ }^{1,2}-$ CUI, R. ${ }^{1,2}-$ ZHANG, X. ${ }^{1,2}-$ FENG, Q. ${ }^{1,2}-$ LIU, S. ${ }^{1,2^{*}}-$ XIE, Z.$^{3^{*}}$ \\ ${ }^{1}$ College of Resources and Environment, Jilin Agricultural University, Changchun 130118, \\ China
}

${ }^{2}$ Key Laboratory of Sustainable Utilization of Soil Resources in Commodity Grain Base of Jilin Province, Changchun 130118, China

${ }^{3}$ College of Plant Science, Jilin University, Changchun, 130062, China.

${ }^{\top}$ These authors contributed equally.

*Corresponding authors

e-mail: liushuxia2005824@163.com,xiezl@jlu.edu.cn

(Received 21 $1^{\text {st }}$ Aug 2021; accepted $23^{\text {rd }}$ Nov 2021)

\begin{abstract}
This study explored the effects of drought stress on the growth and characteristics physiological of different maize varieties. Three maize varieties with different levels of drought tolerance (strong drought tolerance JQ707, medium drought tolerance ND19 and weak drought tolerance FD16) were selected as test crops in a plot experiment while artificial rain shelters were used to control moisture and drought stress. This study was carried out under different degrees of drought stress at the ear stage of maize (Normal water supply, CK; mild drought stress, C1; moderate drought stress, C2; severe drought stress, C3). The results show that drought stress will significantly affect the growth and physiological characteristics of maize tassels. Among them, the anti-oxidant enzyme activity and the content of osmotic adjustment substances in the maize tassels of JQ707 reach the peak value at C2 treatment, which is used to resist the phenomenon of plant water shortage. The anti-oxidant system and osmotic adjustment ability of FD16 maize tassels were poor, and the external morphological structure was also relatively short. This study also found that appropriate stress is beneficial to promote the growth and development of maize tassels and the physiological reaction process. The conclusions of experiment can provide a certain theoretical basis for drought resistance and yield of maize planting in Northeast China.
\end{abstract}

Keywords: water stress, maize tassels, morphological traits, physiological traits

\section{Introduction}

Crop growth and development will be limited by environmental factors, such as drought is considered to be one of the important factors (Fathi and Barari, 2016). Maize (Zea mays L.) is not only one of the most widely planted crops in the world, but also an important food crop and economic crop in China. Northeast China is the main maize producing area in China. In recent years, the trend of drought in Northeast China became more and more serious, which poses a serious threat to maize yield (Yang et al., 2015; Zhang et al., 2011; Fu et al., 2020). The growth cycle of maize can be divided into seedling stage, ear stage, and flowering stage. The ear stage is the key period of maize water demand, and water deficit in this period will affect maize yield to a great extent (Jia et al., 2020; Li et al., 2019). As an important reproductive organ of maize, the development of tassel is closely related to the yield of maize. The tassels of a different maize varieties showed different adaptability to different arid environments, it is specifically reflected in the morphological structure and physiological function of tassels (Xu et al., 2012; Gage et al., 2018). Under drought stress, maize tassels will adapt to 
drought stress through changes in morphology, antioxidant enzymes and osmotic regulation substances accumulation. The development of tassels plays an important role in coordinating the development of male and female tassels, even increasing grain yield in maize (Abdoul et al., 2016; Li et al., 2020). Drought stress will cause the maize tassels to become smaller, and the tassels will be drawn out slowly or even unable to be drawn out (Song et al., 2005). A large number of studies have been done on the effects of drought stress on the physiological characteristics of maize (Zhuang, 2007; Zhang et al., 2012; Liu, 2020; Gill and Tuteja, 2010). For example, maize plants would produce a large number of reactive oxygen species after drought stress (Fathi and Barari, 2016). Reactive oxygen molecules are prone to disrupt the balance of the cell's internal environment. Its excessive presence will lead to lipid reaction of the cell membrane and destroy the structure of protein and enzyme on the cell membrane (Dumanović et al., 2021). To avoid the disturbance of the internal cell environment caused by excessive drought, maize will produce a series of defense mechanisms (Sayadi et al., 2016; Zhao, 2016). The antioxidant system enzymes played a positive role in eliminating excessive oxygen free radicals (Guo et al., 2018); under drought stress, the presence of soluble protein, proline and soluble sugar could maintain the balance of intracellular osmotic pressure and improve the stress resistance of crops, which also play a key role in resisting adversity stress (Tahereh et al., 2011; Blum, 2017; Liu et al., 2011). Therefore, it is of great significance to explore the influence mechanism of different degrees of drought stress on the tassel of different maize varieties.

At present, many studies have demonstrated the effects of drought stress on the growth and physiological traits of maize, most of them, however, only discuss the effects on the growth morphology of maize plants and the physiological characteristics of ear leaves, there are few types of researches on the morphological and physiological characteristics of maize tassels. Therefore, to explore and clarify the effects of curing on tassel growth and physiological characteristics of different maize varieties, this experiment selected three different maize varieties for comparison at the same time and analyzed the morphology and structure of male panicle of different maize varieties, as well as the enzyme activity of the antioxidant system, the content of osmotic regulating substances and the content of malondialdehyde by setting different degrees of drought stress. It will provide a certain theoretical basis for drought resistance and yield protection of maize in Northeast China.

\section{Materials and methods}

\section{Experimental design}

The research was conducted in the Qiqihar Branch of Heilongjiang Academy of

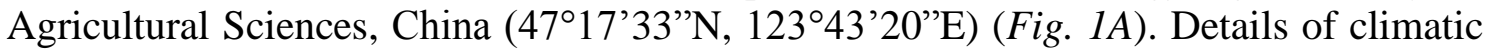
conditions of the maize growth cycle are shown in Figure $1 B$. The general situation of the test site and the growth of maize in the test are shown in Figure 2. The tested soil was chernozem and the basic characteristics of the soil $(0-20 \mathrm{~cm})$ were as follows: $\mathrm{pH}$, 7.82; organic matter, $26.1 \mathrm{~g} \cdot \mathrm{kg}^{-1}$; available nitrogen, $102 \mathrm{~g} \cdot \mathrm{kg}^{-1}$; available phosphorus $15.9 \mathrm{mg} \cdot \mathrm{kg}^{-1}$; available potassium, $130 \mathrm{mg} \cdot \mathrm{kg}^{-1}$.

The tested crop was the three varieties of maize: Jinqing 707 (JQ707) with strong drought tolerance, Nendan 19 (ND19) with medium drought tolerance, and Fudan 16 (FD16) with weak drought tolerance. A total of 12 plots were set up in the experiment, each with an area of $12 \mathrm{~m}^{2}(4 \mathrm{~m} \times 3 \mathrm{~m})$. In order to simulate natural drought conditions, 
open the mobile rain-proof shelter in rainy days to control soil moisture, and close the shelter in sunny days to make maize photosynthesis normally.

The experiment was conducted in maize period different degrees of drought stress, set up to deal with four different moisture gradient, normal water supply as control (CK, keep the soil moisture content is more than $80 \%$ of field capacity), mild drought stress (C1, keep the soil moisture maintained at $70 \%-80 \%$ of field capacity), moderate drought stress (C2, keep the soil moisture maintained at $60 \%-70 \%$ of field capacity) and severe drought stress (C3, keep the soil moisture maintained at 50\%-60\% of field capacity). During the stress period, a soil moisture content analyzer (Instrument model: TZS-1K-G) was used to regularly detect the soil moisture content, and water was supplemented according to the measured moisture content to ensure that the soil moisture content during the stress period was within the set soil moisture content range. A one-time application of fertilizer $30 \mathrm{~kg}$, $\mathrm{N}: \mathrm{P}: \mathrm{K}=22: 14: 6$. Each treatment was repeated three times with a normal water supply as control $(\mathrm{CK})$, and each repeat contains three maize plants.
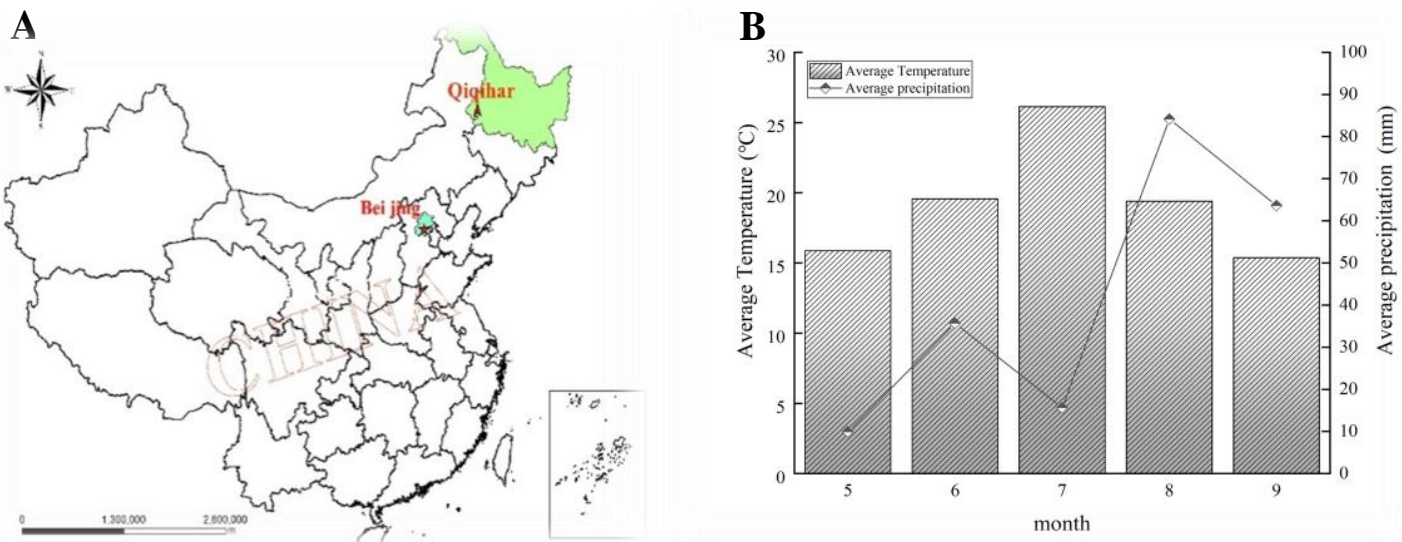

Figure 1. Experimental location and climatic conditions of maize growth cycle
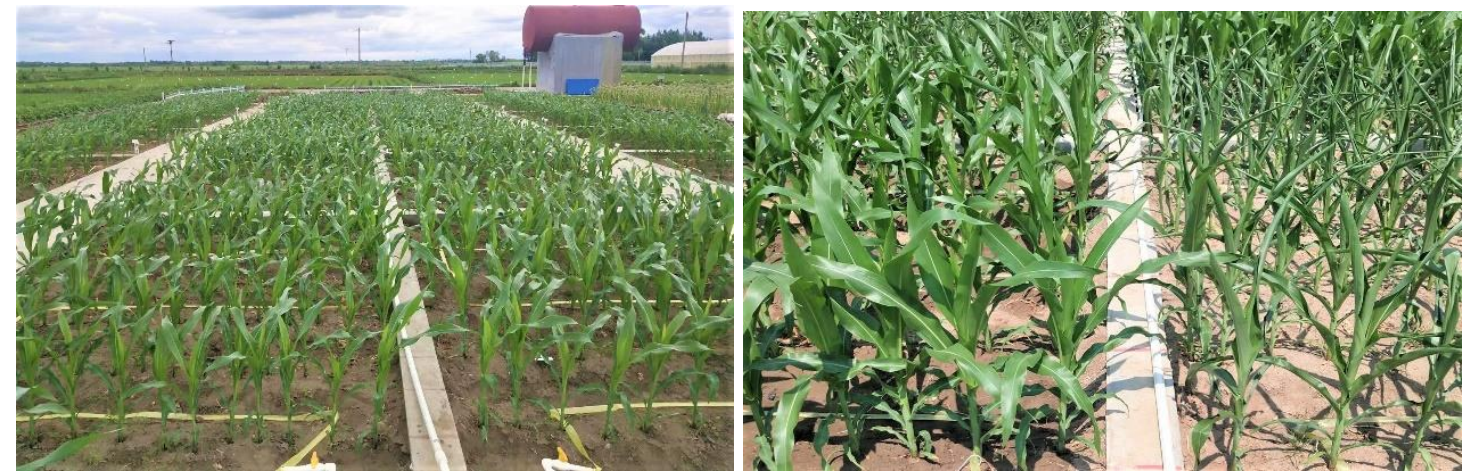

Figure 2. Overview of the test site and the growth of maize in the test

\section{Determination of morphological index in tassel of maize}

Tassel size $=$ tassel spindle length + average branch length $\times$ branching number.

Spindle length of tassel was measured with a tape measure. The spindle diameter is measured with a vernier caliper. Dry matter weight was determined by the drying method. 


\section{Determination of physiological index in tassel of maize}

The enzyme activities of the antioxidant system were detected by the ELISA detection kit. Weigh $1 \mathrm{~g}$ tassel sample, cut it into pieces, put it into a mortar, add $10 \mathrm{ml}$ PBS buffer solution and grind it fully, after centrifugation at $4000 \mathrm{r} \cdot \mathrm{min}^{-1}$ for $15 \mathrm{~min}$, and then take the supernatant. The enzymatic activities of SOD, CAT, and POD in a tassel of maize were determined by an enzymatic plate analyzer. The content of proline was determined by sulfosalicylic acid method; Soluble sugar content was determined by anthrone colorimetry; Soluble protein content was determined by coomassie bright blue method; The content of malondialdehyde was determined by thiobarbituric acid method; The methods were based on Zou (2003).

\section{Data analysis}

All data were produced using Microsoft Excel 2016 software. The experimental data are all used SPSS 21.0 software for one-way statistical analysis of variance, and Origin 2018 software for plotting.

\section{Results}

\section{Effects of drought stress on morphological traits in tassel of different maize varieties}

To explore the effect of drought stress on the morphological structure of the tassel of different maize varieties, the following analysis from four aspects were made: tassel size, spindle length, spindle diameter, and dry matter weight (Table 1).

Table 1. Effects of drought stress on the morphological structure of tassels of different maize varieties

\begin{tabular}{c|c|c|c|c|c}
\hline Treatment & Maize variety & $\begin{array}{c}\text { Tassel size } \\
(\mathbf{c m})\end{array}$ & $\begin{array}{c}\text { Spindle length } \\
(\mathbf{c m})\end{array}$ & $\begin{array}{c}\text { Coarse spindle } \\
(\mathbf{m m})\end{array}$ & $\begin{array}{c}\text { Tassel dry } \\
\text { weight }(\mathbf{g})\end{array}$ \\
\hline \multirow{3}{*}{ CK } & JQ707 & $188.17 \pm 8.61 \mathrm{a}$ & $38.67 \pm 3.06 \mathrm{a}$ & $6.00 \pm 0.50 \mathrm{a}$ & $3.09 \pm 0.08 \mathrm{a}$ \\
& ND19 & $187.70 \pm 13.79 \mathrm{a}$ & $35.67 \pm 2.52 \mathrm{a}$ & $5.93 \pm 0.81 \mathrm{a}$ & $3.14 \pm 0.13 \mathrm{a}$ \\
& FD16 & $156.60 \pm 7.86 \mathrm{~b}$ & $35.33 \pm 1.53 \mathrm{a}$ & $6.30 \pm 0.82 \mathrm{a}$ & $2.98 \pm 0.06 \mathrm{a}$ \\
\hline \multirow{3}{*}{ C1 } & JQ707 & $179.33 \pm 10.68 \mathrm{a}$ & $35.19 \pm 0.76 \mathrm{a}$ & $4.83 \pm 0.76 \mathrm{a}$ & $3.23 \pm 0.22 \mathrm{a}$ \\
& ND19 & $178.79 \pm 8.28 \mathrm{a}$ & $31.33 \pm 2.93 \mathrm{a}$ & $5.17 \pm 0.29 \mathrm{a}$ & $3.21 \pm 0.08 \mathrm{a}$ \\
& FD16 & $143.68 \pm 12.71 \mathrm{~b}$ & $32.17 \pm 0.76 \mathrm{a}$ & $4.83 \pm 0.29 \mathrm{a}$ & $3.03 \pm 0.02 \mathrm{~b}$ \\
\hline \multirow{3}{*}{ C2 } & JQ707 & $163.42 \pm 3.85 \mathrm{a}$ & $35.05 \pm 0.93 \mathrm{a}$ & $5.00 \pm 0.87 \mathrm{a}$ & $2.53 \pm 0.02 \mathrm{a}$ \\
& ND19 & $153.91 \pm 12.06 \mathrm{a}$ & $31.17 \pm 0.76 \mathrm{~b}$ & $4.37 \pm 0.71 \mathrm{a}$ & $3.02 \pm 0.03 \mathrm{a}$ \\
& FD16 & $121.81 \pm 6.10 \mathrm{~b}$ & $31.51 \pm 0.77 \mathrm{~b}$ & $4.50 \pm 0.50 \mathrm{a}$ & $2.21 \pm 0.27 \mathrm{a}$ \\
\hline \multirow{3}{*}{ C3 } & JQ707 & $148.36 \pm 7.29 \mathrm{a}$ & $34.83 \pm 0.76 \mathrm{a}$ & $4.67 \pm 0.76 \mathrm{a}$ & $2.28 \pm 0.02 \mathrm{a}$ \\
& ND19 & $137.23 \pm 12.97 \mathrm{a}$ & $31.23 \pm 0.71 \mathrm{~b}$ & $4.43 \pm 0.81 \mathrm{a}$ & $2.89 \pm 0.12 \mathrm{a}$ \\
& FD16 & $111.37 \pm 13.86 \mathrm{~b}$ & $29.50 \pm 0.50 \mathrm{c}$ & $4.13 \pm 1.21 \mathrm{a}$ & $1.89 \pm 0.08 \mathrm{a}$ \\
\hline
\end{tabular}

Data in the table are mean \pm standard deviation, and different lowercase letters after data in the same column indicate significant difference $(\mathrm{P}<0.05)$

As can be seen from Table 1, with the increase of drought stress, the tassel size of different maize varieties decreased to different degrees, tassel spindle length, and diameter of tassel spindle shortened to different degrees, and the effects of drought on 
different varieties were different. Among all the drought stress treatments, JQ707 was the least affected. Under normal water supply, the tassel size of FD16 was smaller than that of JQ707 and ND19, but there was no significant difference in the length and diameter of its main axis. Under severe drought stress, the tassel size, spindle length and spindle diameter of the tested maize varieties were the lowest values relative to CK. The main shaft length of the tassel of JQ707, ND19, and FD16 was shortened by $11.02 \%$, $14.22 \%$, and $19.76 \%$, and the main shaft diameter of the tassel was reduced by $28.48 \%$, $33.86 \%$, and $52.54 \%$, respectively.

With the increase of drought stress, the dry matter weight of different maize tassels showed a trend of first increasing and then decreasing. Mild drought stress can promote the accumulation of dry matter in tassels. Compared with $\mathrm{CK}$, the dry matter weight of JQ707, ND19, and FD16 increased by $4.33 \%, 2.18 \%$, and $1.65 \%$, respectively. Moderate and severe drought stress was not conducive to dry matter accumulation in the tassels of maize. The dry matter weight of the three varieties of maize decreased compared with that of $\mathrm{CK}$. The dry matter accumulation was the order of ND19 > JQ707 > FD16.

\section{Effects of drought stress on physiological characteristics of different maize varieties}

Effects of drought stress on enzyme activity of antioxidant system and malondialdehyde content in tassel of different maize varieties

To investigate the effects of drought stress on the contents of antioxidant enzymes and MDA in a different tassel of maize, the following analysis was conducted: as shown in Figure 2A, there was no significant difference in SOD content of tassel under different drought stress, but SOD had a higher content in tassels. The SOD content in the tassels of the weak drought-tolerant variety FD16 can be maintained above $800 \mathrm{U} \cdot \mathrm{g}^{-1}$, and the content of strong drought-tolerant variety JQ707 and medium drought-tolerant variety ND19 can be maintained above $1000 \mathrm{U} \cdot \mathrm{g}^{-1}$. This indicates that SOD plays an important role in the tassel of maize, and its role is to decompose excessive $\mathrm{O}^{2} \cdot$ in the body so as to prevent the subsequent formation of hydrogen peroxide and hydroxyl radicals. The change of SOD content can also indicate that its secretion level did not change significantly with the degree of drought. The enzyme activity of FD16 was the highest under moderate drought stress, which was $22.95 \%$ higher than that of CK, and its activity returned to the level of CK under severe drought stress. Under moderate drought stress, the enzyme activities of JQ707 and ND19 were $29.38 \%$ and $22.80 \%$, which were higher than those of CK, respectively, and under severe drought stress, the enzyme activities were also $9.67 \%$ and $6.66 \%$, which were also higher than those of CK, respectively. Therefore, we can see the difference of SOD activity in tassel between drought-tolerant maize and drought-resistant maize under drought conditions.

As shown in Figure $2 B$ and $C$, the activity change trend of CAT and POD is different from SOD. The activities of CAT and POD were significantly affected by drought stress. The overall trend of CAT is that the enzyme activity reaches its peak under moderate drought stress and decreased under severe drought stress. Under mild and moderate drought stress, the most significant change was CAT in JQ707 and ND19, the CAT secretion of them was more than twice that CK treatment, and under severe drought stress, it was also increased by $38.45 \%$ and $35.92 \%$, respectively. However, for the weak drought-tolerant variety FD16, the changes in CAT activity in 
its tassels are not significant. The activity of SOD under mild drought stress was $36.04 \%$ higher than that of CK, while its secretion volume under severe drought stress was not significantly different from that of CK. The reason why the content of CAT in the tassels changes so significantly was probably due to the excessive formation of superoxide free radicals in the tassel cells under drought conditions and the secretion of SOD did not change much. Therefore, excessive oxygen free radicals will induce the production of $\mathrm{H}_{2} \mathrm{O}_{2}$, and the maize tassels will secrete excessive CAT to eliminate $\mathrm{H}_{2} \mathrm{O}_{2}$. The POD exists in the CAT enzyme body, the secretion of CAT increased and POD also increased, and the mode of action of POD was to use hydrogen peroxide as the electron acceptor to catalyze the oxidation of the substrate, thereby, removing harmful substances produced in plants due to adversity, such as hydrogen peroxide, oxidized phenols, and amines. This also indirectly explained the drought tolerance of the three maize varieties.
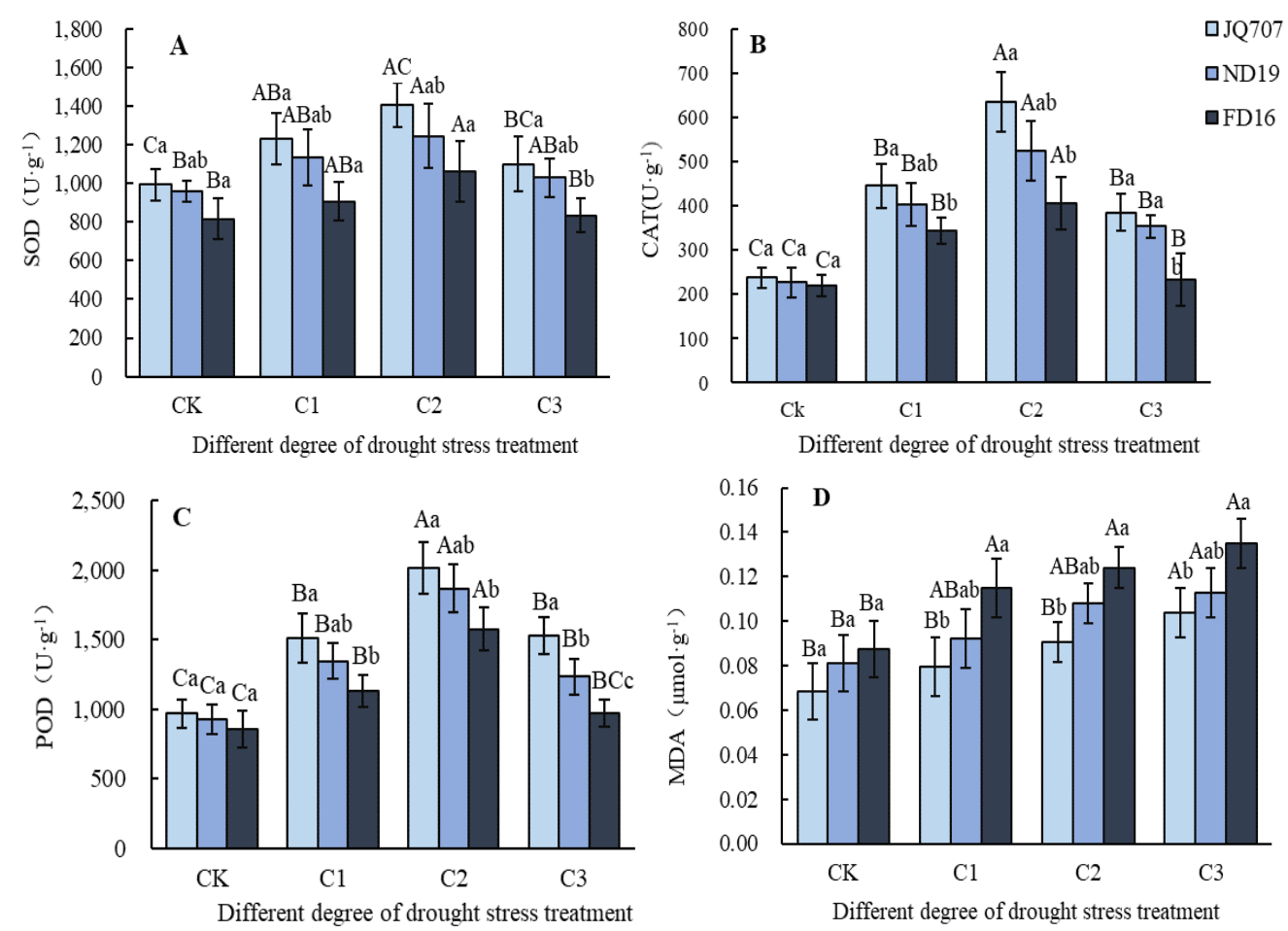

Figure 2. Effects of drought stress on the activities of antioxidant enzymes (SOD, POD and $C A T)$ and MDA content in tassels of different maize varieties. The error column shown in the figure is the positive and negative deviation of the standard deviation of antioxidant enzyme data of different maize tassels under the same drought treatment. Capital English letters represent the significant difference of antioxidant enzyme activity between different drought stress treatments under the same maize variety, and small letters represent the significant difference of antioxidant enzyme activity data of different maize tassels under the same drought stress treatment

As shown in Figure 2D, the content of MDA increased significantly with the increase of drought. The growth rates of JQ707, ND19, and FD16 were 11.8\%, $11.1 \%$, and $15.2 \%$, respectively. Under severe drought stress, the MDA content in the tassels of the three maize varieties all reached a peak. The MDA content of FD16 increased by $35.16 \%$ compared with CK under severe drought stress, and the secretion of FD16 
increased significantly compared with JQ707 and ND19. This was because the secretion of SOD, CAT, and POD in the tassel of FD16 returned to a low level under severe drought stress, unable to decompose too many harmful substances, resulting in a sharp increase in MDA content, while the changing trend of JQ707 and ND19 was less obvious. Since MDA can be used as a criterion for judging the degree of cell damage, the change in its content can show the stress resistance of maize ears, which also proves to a certain extent that these two maize varieties, JQ707 and ND19, have strong resistance to stress.

\section{Effects of drought stress on the content of osmotic regulating substances in tassel of different maize varieties}

In order to explore the effect of drought stress on the osmotic mechanism of different maize tassels, the following analysis was conducted: as shown in Figure 3A, the specific expression of Pro secretion in a tassel of maize was as follows: the growth rate of Pro in tassels of JQ707 and FD16 was higher under mild drought, which increased by $20.8 \%$ and $17.73 \%$ compared with $\mathrm{CK}$, respectively, and slightly lower at moderate levels, while the changing trend of ND19 was opposite to the two. Under severe drought stress, the secretion rate of Pro in the three maize tassels all decreased, but the secretion of JQ707 was still the highest among the three. The reason was probably that the regulation mechanism of proline in different maize tassels was different under mild and moderate drought. JQ707 and FD16 had higher Pro secretion in the early drought period. These maize varieties paid more attention to osmotic pressure regulation in the early drought period so that they could pass through the middle drought period smoothly, while ND19 paid more attention to the regulation in the middle drought period. Under severe drought, ND19 and FD16 increased tassel cell damage and decreased Pro secretion, but the Pro secretion of JQ707 did not decrease but increased, which proved its strong drought resistance to a certain extent.

As shown in Figure 3B, under mild and moderate drought stress, the accumulation of SP in the tassels of the three varieties increased rapidly, and the secretion growth rate reached $60 \%, 37 \%$, and $32 \%$, respectively, while under severe drought, the secretion rate of the three varieties all decreased. Although the SP secretion rate in FD16 tassels under severe drought exceeded $20 \%$, the SP content in tassels was only $5.67 \mathrm{mg} \cdot \mathrm{g}^{-1}$, which was the same as that of JQ707 under mild drought. This also proved the reason for the strong drought resistance of JQ707.

As shown in Figure 3C, the change of SS content in tassels also increased with the increase of drought degree, but there was no significant difference among the treatments. Compared with JQ707 and ND19, the secretion rate of SS in tassels of FD16 was always low, and its highest content was only $3.35 \mathrm{mg} \cdot \mathrm{g}^{-1}$, which was equivalent to the content of JQ707 and ND19 under mild and moderate drought, indicating that the secretion ability of SS in maize tassel of FD16 was poor. Under mild drought, SS accumulation in male panicles of ND19 was higher, but under moderate and severe drought, SS secretion rate in male panicles of ND19 was significantly reduced, which indicated that SS regulation ability of ND19 was stronger under mild drought. For all treatments, the accumulation of SS in the tassels of JQ707 was at a higher level. Under severe drought stress, the SS secretion rate in the tassels was 3-4 times higher than that of ND19 and FD16, and the SS content in the tassels could reach $4.22 \mathrm{mg} \cdot \mathrm{g}^{-1}$. This showed that JQ707 SS had a strong secretion ability and good stress resistance. 

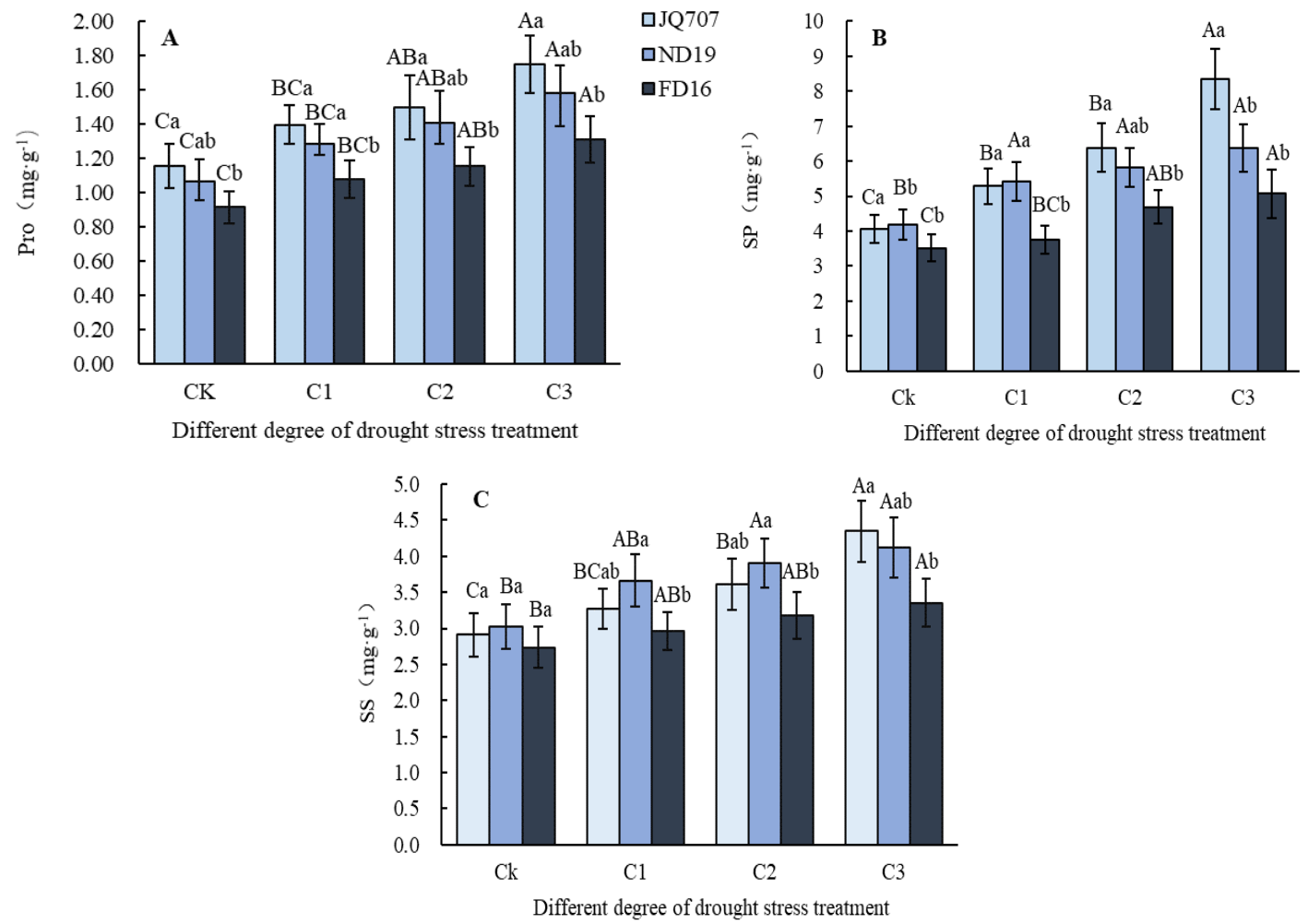

Figure 3. Effect of drought stress on osmotic adjustment substances (Pro, SP and SS) in tassels of different maize varieties. The error column shown in the figure is the positive and negative deviation of the standard deviation of osmotic adjustment substances data of different maize tassels under the same drought treatment. Capital English letters represent the same maize variety, and the contents of osmotic adjustment substances in different drought stress treatments are significantly different, while small letters represent the same drought stress treatment, and the contents of osmotic adjustment substances in different maize tassels are significantly different

\section{Discussion}

In this experiment, the poor performance of weak drought-tolerant varieties was due to the poor secretion ability of antioxidant enzymes compared with the other two varieties, and during severe drought, the antioxidant system enzymes in the tassels cannot be excessively secreted, their secretions return to normal levels, unable to eliminate the excessive harmful substances produced in the tassels. Medium droughttolerant varieties have higher antioxidant system enzyme activity than weak droughttolerant varieties, which can decompose excessive oxygen free radicals and harmful substances in the tassels to improve the stress resistance of the tassels. In all treatments, the strong drought-tolerant varieties have higher secretion of antioxidant system enzymes and strong cell activity, and the tassels have strong stress resistance and perform well under drought stress. Previous studies in other plants have also found that drought-tolerant plant varieties have a strong antioxidant enzyme system to effectively control the content of active oxygen in the body (Hou et al., 2018; Zhang et al., 2018; Xu et al., 2014).

The possible mechanism of the antioxidant system in maize tassels as shown in Figure 4A: under drought conditions, maize cells will lose water, then it will produce a 
large number of reactive oxygen molecules. These oxygen free radicals can cause oxidative damage to the cell membrane, induce its lipidation, and produce a large amount of malondialdehyde (MDA). A large number of reactive oxygen species and MDA could change the fluidity and permeability of cell membranes. Finally, damage the structure and function of cells. This excessive damage encourages the endocrine of the maize tassel to secrete antioxidant system enzymes (such as SOD, POD, and CAT) to eliminate this side effect. For example, SOD eliminates $\mathrm{O}^{2-i n}$ the cell, thereby generating $\mathrm{H}_{2} \mathrm{O}$ and $\mathrm{O}_{2}$, which can avoid subsequent oxidation reactions. The POD and CAT mainly eliminate the $\mathrm{H}_{2} \mathrm{O}_{2}$ molecules in the maize tassel and also produce water and $\mathrm{O}_{2}$, reducing the active oxygen content in the tassels to a balanced state, and the water and oxygen produced will be used by the tassels, which can compensate to a certain extent. In this way, the damage caused by drought can be compensated, and then the resistance of tassels can be enhanced.

Combined with the analysis of the mechanism of osmotic adjustment, the reason for the poor performance of weak drought-tolerant varieties under drought conditions is that the secretion ability of tassel osmotic adjustment substances is reduced and the overall content is less. Compared with the weak drought tolerant varieties, the osmotic adjustment ability of the medium drought-tolerant tassel was stronger, and the osmotic adjustment substance content in the tassel increased significantly under mild and moderate drought, but the secretion rate decreased under severe drought. The reason for strong drought-tolerance of tassel of maize may be that: (1) In the early period of drought, the internal osmotic adjustment substance accumulation in the tassel is sufficient, so the secretion rate in the later period was slow; (2) Due to severe drought, the cells lose water and their activity decreases, resulting in a decrease in the secretion rate of osmotic substances.

In this study, the change of osmotic adjustment substance content and the changing trend of antioxidant system enzymes are basically the same. This indicates that osmotic regulation can protect the enzyme activity that is beneficial to the removal of active oxygen under drought conditions, which is basically consistent with the results of previous studies (Xu et al., 2014).

The possible mechanism of osmotic adjustment in maize tassels is shown in Figure 4B: under drought conditions, maize cannot normally absorb water from the soil and lose water, which leads to the inconsistency of osmotic pressure inside and outside the cell and further leads to cell shrinkage and activity reduction. Under this condition, a large number of osmotic adjustment substances (such as pro, SS, and SP) will be secreted and accumulated inside the tassel, so as to reduce the cell water potential and maintain the same osmotic pressure inside and outside the cell. Finally, the tassel can grow normally and complete the late pollination.

Although maize tassels can improve their drought resistance by secreting antioxidant enzymes and osmotic adjustment substances, the peroxidation and osmotic imbalance caused by drought still have a serious impact on the external morphological structure of maize tassels. The results showed that the ability of antioxidant system enzyme and osmotic adjustment substance secretion of weak drought-tolerance maize tassel was weak, and its stress resistance was poor. Therefore, compared with the other two kinds of drought-tolerant maize, the development of weak drought tolerant maize tassels is slower, and the length of tassels and the number of branches are shortened most significantly. This is similar to the previous results (Jia et al., 2019). 


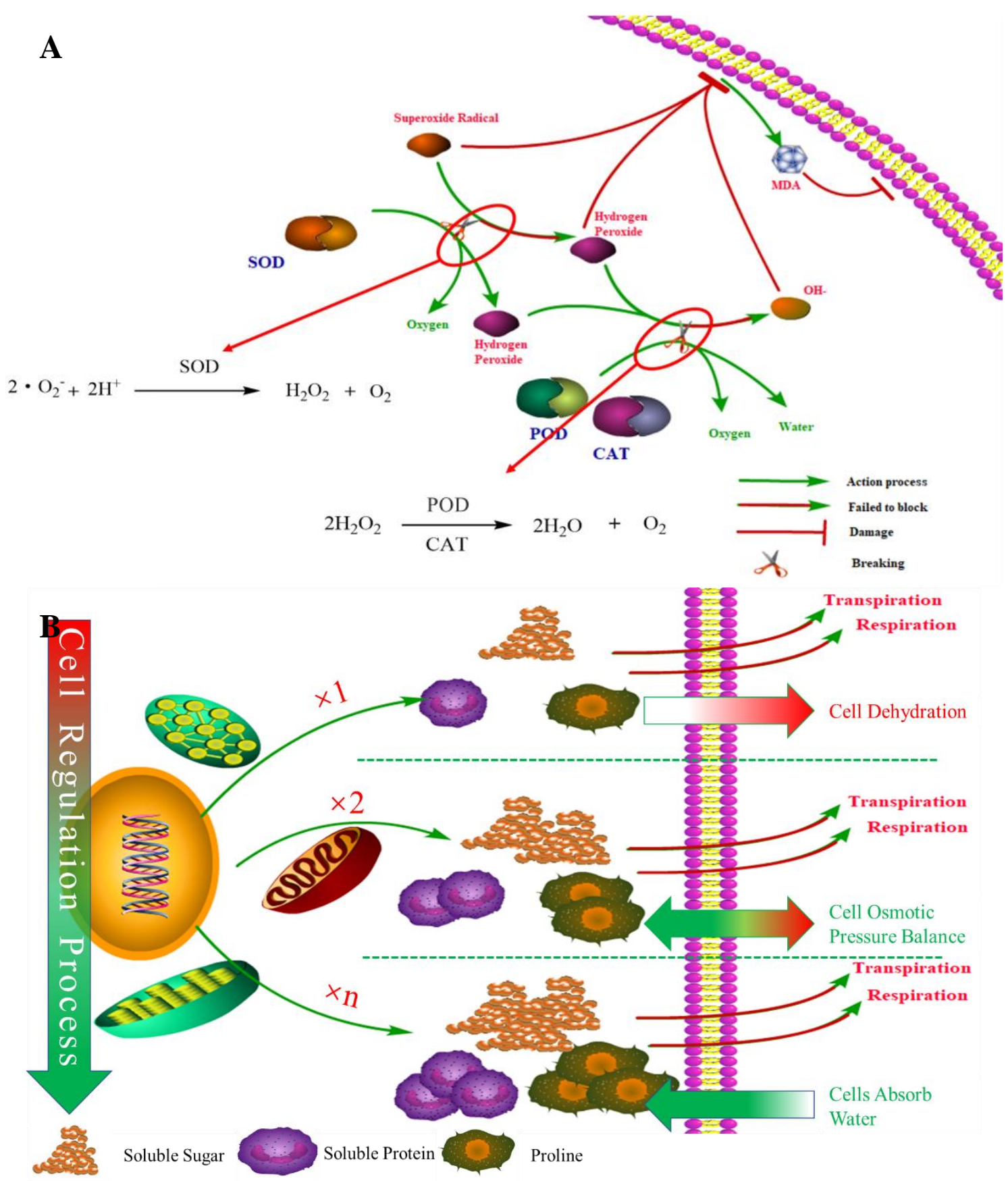

Figure 4. Schematic diagram of physiological function in maize under drought stress. (A)

Schematic diagram of the physiological effects of antioxidant system enzymes and malondialdehyde in maize ears under drought. (B) Schematic diagram of the physiological effects of osmotic adjustment substances in maize ears under drought

In conclusion, from the analysis of drought degree, appropriate drought is conducive to the normal development of maize tassels, and can promote the secretion of antioxidant enzymes and osmotic adjustment substances. However, excessive drought or prolonged drought stress are not conducive to the development of tassels, which will cause excessive accumulation of harmful substances in maize tassels and irreversible damage to maize tassels. From the analysis of drought tolerance of maize varieties, 
under drought stress, the internal physiological response of maize tassels with strong drought tolerance will be more active to resist the harmful substances produced by drought stress. The maize varieties with weak drought tolerance not only have weak internal physiological response of tassels, but also have a greater impact on the external morphological structure.

\section{Conclusion}

The study found that drought stress would seriously affect the growth and physiological characteristics of maize tassels. The results showed that the strong drought-tolerant varieties had a strong antioxidant enzyme system and osmotic adjustment ability. The changes of antioxidant enzyme activity and osmotic adjustment substance content in tassel were consistent, which indicated that osmotic adjustment could coordinate the protective enzyme activity to a certain extent. Under severe drought conditions, there was a negative correlation between the activity of antioxidant enzymes and the content of MDA, which indicated that MDA in tassels would destroy the stability of membrane structure and hinder the secretion of antioxidant enzymes. Maize tassel is an important reproductive organ of maize. Therefore, this study suggests that drought tolerant varieties suitable for local climate should be selected in the process of maize planting, and appropriate drought stress can be carried out. In maize breeding, we can consider increasing the density of SOD regulatory genes in maize to improve the drought resistance of maize.

Acknowledgements. We thank two anonymous reviewers for their comments on an earlier draft of this manuscript.

Data availability. The data used in this study is available from the corresponding author upon request.

Funding. This work is a contribution Jilin Provincial Key Research and Development Program (20200403069SF); Cultivation Project of "Interdisciplinary Integration and Innovation" of Jilin University: The research on the integration innovation system of Rural Revitalization promoting the protection of black land, agricultural transformation and income multiplication in Northeast China.

\section{REFERENCES}

[1] Blum, B. (2017): Osmotic adjustment is a prime drought stress adaptive engine in support of plant production. - Plant, Cell Environment 40(1).

[2] Dumanović, J., Nepovimova, E., Natić, M., Kuča, K., Jaćević, V. (2021): The Significance of reactive oxygen species and antioxidant defense system in plants: a concise overview. - Frontiers in Plant Science. https://doi.org/10.3389/fpls.2020.552969.

[3] Fathi, A., Barari, D. (2016): Effect of drought stress and its mechanism in plants. International Journal of Life Sciences 10(1).

[4] Fu, C., Zhang, Y., Mi, N., Ming, H., Zhang, S., Zhang, H., Zhao, X. (2020): Maize (Zea mays L.) physiological responses to drought and rewatering, and the associations with water stress degree. - Agricultural Water Management 241.

[5] Gage, J. L. (2018): Tassel Morphology in Zea Mays: Novel Phenotyping Tools and Signatures of Selection. - The University of Wisconsin, Madison.

[6] Gill, S. G., Tuteja, N. (2010): Reactive oxygen species and antioxidant machinery in abiotic stress tolerance in crop plants. - Plant Physiology and Biochemistry 48(12). 
[7] Guo, Y. Y., Liu, J., Zhu, Y. L., Bai, Y. W., Li, H. J., Xue, J. Q., Zhang, R. H. (2018): The response of maize leaf photosynthesis and antioxidant enzyme activities to drought stress. - Plant Physiology 54(12): 1839-1846.

[8] Hou, J. (2018): Physiological Mechanism of Different Drought-Resistant Wheat Varieties in Response to Drought Differences. - The Agricultural University of Henan, Henan.

[9] Jia, S., Wang, N., Hao, X., Zong, Y., Zhang, D., Li, P. (2019): Effects of different drought stress treatments on growth and stress physiology of Soybean. - Acta Agriculturae Sinica 34(05): 137-144

[10] Jia, S. J., Li, H. W., Jiang, Y. P., Zhao, G. Q., Wang, H. Z., Yang, S. J., Yang, Q. H., Guo, J. M., Shao, R. X. (2020): Effects of drought stress on leaf photosynthetic characteristics and ear development characteristics of maize. - Acta Ecologica Sinica 40(03): 854-863.

[11] Li, W., Hao, Z., Pang, J., Zhang, M., Wang, N., Li, X., Li, W., Wang, L., Xu, M. (2019): Effect of water-deficit on tassel development in maize. - Gene 681

[12] Li, H. W., Jiang, Y. P., Jia, S. J., Zhao, G. Q., Wang, Y. C., Yang, Q. H., Liu, T. X., Li, C. H., Shao, R. X. (2020): Advances in studies on effects of drought stress on ear development of maize. - Journal of Maize Science 28(02): 90-95.

[13] Liu, C., Liu, Y., Guo, K., Fan, D., Li, G., Zheng, Y., Yu, L., Yang, R. (2011): Effect of drought on pigments, osmotic adjustment and antioxidant enzymes in six woody plant species in karst habitats of southwestern China. - Environmental and Experimental Botany 71(2).

[14] Liu, G. (2020): Physiological and Biochemical Characteristics of Drought Resistance in Maize and Analysis of Differentially Expressed Genes. - The Agricultural University of Hebei, Hebei.

[15] Sayadi, A., Zou, M., Tu, J., Qiu, J., Liu, Z. (2016): Breeding for drought tolerance in maize (Zea mays L.). - American Journal of Plant Sciences 7(14)

[16] Song, F. B., Dai, J. Y. (2005): The response and adaptability of maize to drought stress II . The growth and development of maize ears and tassels in response to drought stress. Journal of Jilin Agricultural University 01: 1-5 + 10 .

[17] Tahereh, Z.-A., Naser, B., Mohammad, S.-H. (2016): Physiological and morphological response to drought stress in seedlings of ten citrus. - Trees 30(3).

[18] Xu, H. W., Song, F. B., Tong, S. Y. (2012): Advances in development and physiological characteristics of male and female ears in maize. - Guangdong Agricultural Sciences 39(03): 22-24.

[19] Xu, H., Lu, Y., Xie, Z., et al. (2014): Changes in nitrogen metabolism and antioxidant enzyme activities of maize tassel in black soils region of northeast China. - Frontiers in Plant Science 5.

[20] Yang, R. Z. (2015): Temporal and spatial characteristics and risk comprehensive assessment of main agrometeorological disasters of maize in Northeast China. - PhD Dissertation, Chinese Academy of Meteorological Sciences.

[21] Zhang, S. J., Zhang, Y. S., Ji, R. P., Cai, F., Wu, J. W. (2011): Analysis of Distributional Characteristics and Primary Causes of Maize Drought in Northeast China 29(01): 231236.

[22] Zhang, R., Guo, D., Zhang, X., Lu, H., Liu, J., Li, F., Hao, Y., Xue, J. (2012): The effect of drought stress during the spinning period on maize physiological characteristics and material production. - Acta Agronomica Sinica 38(10): 1884-1890.

[23] Zhang, C., Shi, S., Wu, F. (2018): Effects of drought stress on root growth and physiological characteristics of different drought-resistant alfalfa varieties. - Chinese Agricultural Sciences 51(05): 868-882.

[24] Zhao, W. S. (2016): Response of stomatal development of maize leaves to drought and effects of repeated drought on photosynthesis and drought resistance of maize. - $\mathrm{PhD}$ Dissertation, Northwest A \& F University. 
[25] Zhuang, Y. L. (2007): Effects of drought on gene expression of male and female ears of maize and cloning and analysis of glutamate decarboxylase gene. - PhD Dissertation, The University of Shandong.

[26] Zou, Q. (2003): Experimental Guidance of Plant Physiology. - China Agricultural Press, Beijing. 